\title{
The v-erbA Oncoprotein of the AEV Transforming Retrovirus Binds to the Promoter Region of the Erythroid- specific Band 3 Gene
}

\author{
SIGURDUR INGVARSSON" \\ Laboratory of Cell Biology \\ Department of Pathology \\ National University Hospital \\ P. O. Box 1465 \\ I2I Reykjaik, leeland \\ BJÖRN VENNSTRÖM \\ Karolinska Institutet \\ Department of Molecular Biology \\ Box 60400 \\ S-l040l Stockholm, Sweden
}

\begin{abstract}
Avian erythroblastosis virus (AEV) which carries two oncogenes, v-erbA and $v$ erbB. causes erythroleukemia and sarcomas in young chickens and transforms embryo fibroblasts and bone marrow cells in culture. The v-erbA oncogene encodes an aberrant version of a gene for a nuclear thyroid hormone receptor (cerbA, TR) and functions in neoplasia by blocking erythroid differentiation and by altering the growth properties of tibroblasts. Owing to the multiple point mutations and a small C-terminal deletion, v-erbA has lost the ability to bind $\mathrm{T} 3$, but still binds to DNA in a sequence-specific fashion. The phenotypic effects of $v$-erbA are correlated with and probably caused by $v$-erbA-mediated repression of a set of erythrocyte-specific genes, but their expression seems to be required during normal erythrocyte maturation. In differentiating erythroblasts the v-erbA oncoprotein specifically arrests expression of the avian erythrocyte anion transporter (band 3), CAll and ALA-S genes at the transcriptional level.' Overexpression of the TR is required to modulate erythrocyte-specific gene expression in the presence of $T 3$. The $v$-erbA oncogene might act as a constitutively active repressor of erythrocyte gene transcription, having lost its ability to depress (or activate) these genes in response to $\mathrm{T} 3$ or even retinoic acid.

To determine whether erbA gene products and related receptors are able to bind the regulatory region of the band 3 gene we performed in vitro McKay assays (co-immunoprecipitation $)^{2}$ and band shifts. Nuclear extracts derived from HeLa cells overexpressing erbA proteins in a vaccinia virus expression vector were used. ${ }^{3}$ End-labeled band 3 gene fragments were incubated with $v$-erbA containing
\end{abstract}

"Author to whom correspondence should be addressed: Tel: 354-1-601906: FAX: 354-1601943. 
nuclear extracts in the presence of increasing amounts of unlabeled competitors. and subsequently subjected to immunoprecipitation using anti-erbA. FICURE 1 shows an analysis of the co-precipitating fragments under various conditions of stringency. The 383 bp Rsal (including one of the promoters and the first exon) and $1.1 \mathrm{~kb} \mathrm{Rsal}$ (the 5' region of the gene) fragmentst were preferentially precipitated from erbA containing extracts (FIG. 1). The 383 bp was mapped further with gel retardation assays on smaller fragments and synthesized oligos (FIG. 2 and data not shown).

A synthesized oligonucleotide of 43 bp from a sequence in the 3 ' part of the first exon, at position 163-192, with a sequence similar to core thyroid hormone receptor element (TRE) and including a retinoic acid receptor element (RARE) half site ${ }^{5}$ was shifted in mobility (FIG, 2). Binding of the oligonucleotide within $\mathrm{TR} \alpha$ nuclear extract led to the appearance of a triplet of three bands exhibiting lower mobility than the free DNA. When the dir2 or dir4 oligonucleotides (that
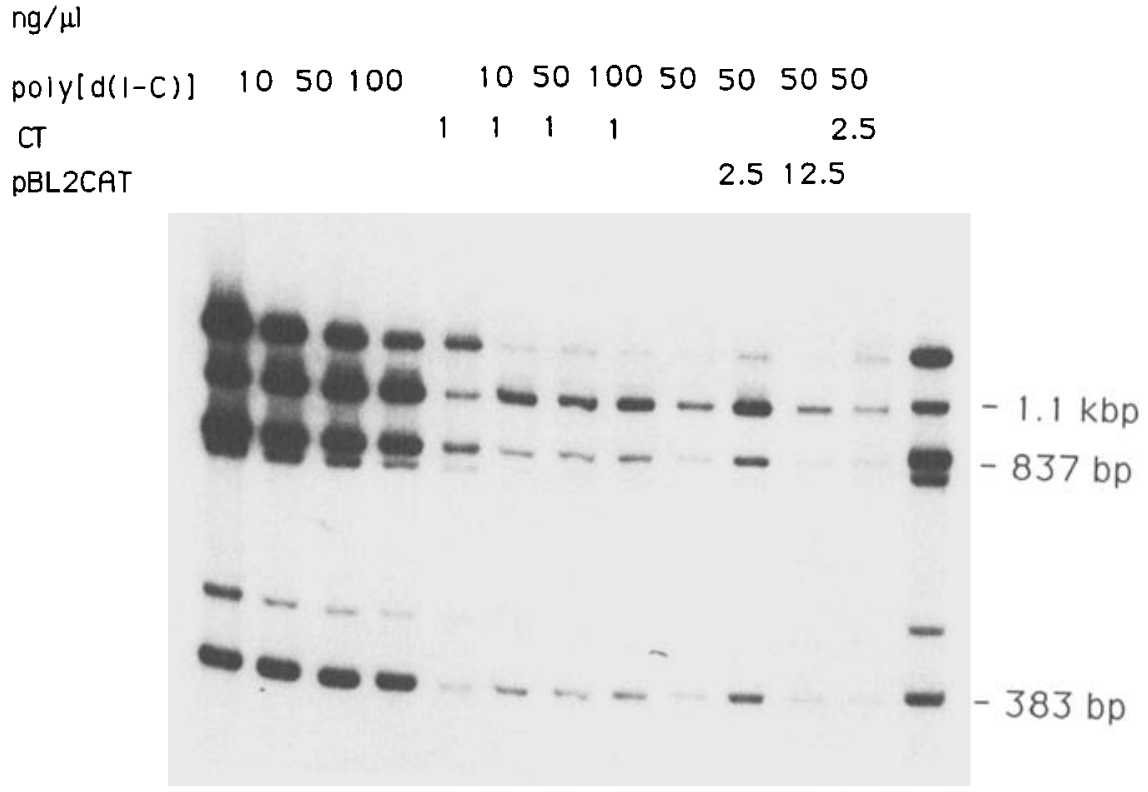

$$
\text { CO.I.P. - VEA PBIII23ER }
$$

FIGURE 1. Co-immunoprecipitation of the chicken band 3 gene fragments with the v-erbA protein. The co-immunoprecipitation assays were performed according 10 McKay 1981. ${ }^{2}$ A plasmid containing a $2.3 \mathrm{~kb}$ genomic clone of the band 3 gene ${ }^{+}$was cleaved with Rsal and EcoRI (pBIII23ER), the fragments were blunted with Klenow enzyme and end-labeled with polynucleotide kinase and incubated with $v$-erbA containing extratcts (VEA) in the presence of different amounts of competitor DNA. The nuclear extracts were derived from HeLa cells infected with a recombinant vaccinia virus carrying the $v$-erbA gene. ${ }^{3}$ Complexes were then precipitated with anti-erbA antibodies bound to Staphylococcus proteinA. Cold competitor DNA were various concentrations by poly $[d(\mathrm{I}-\mathrm{C})]$. calf thymus DNA (CT) and the pBL2CAT plasmid. The numbers above the lanes indicate $\mathrm{ng} / \mu \mathrm{l}$ of cold competitor. 


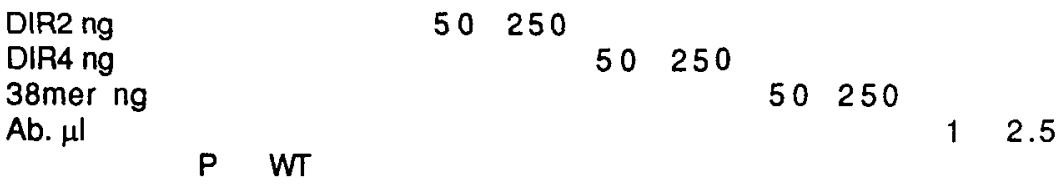

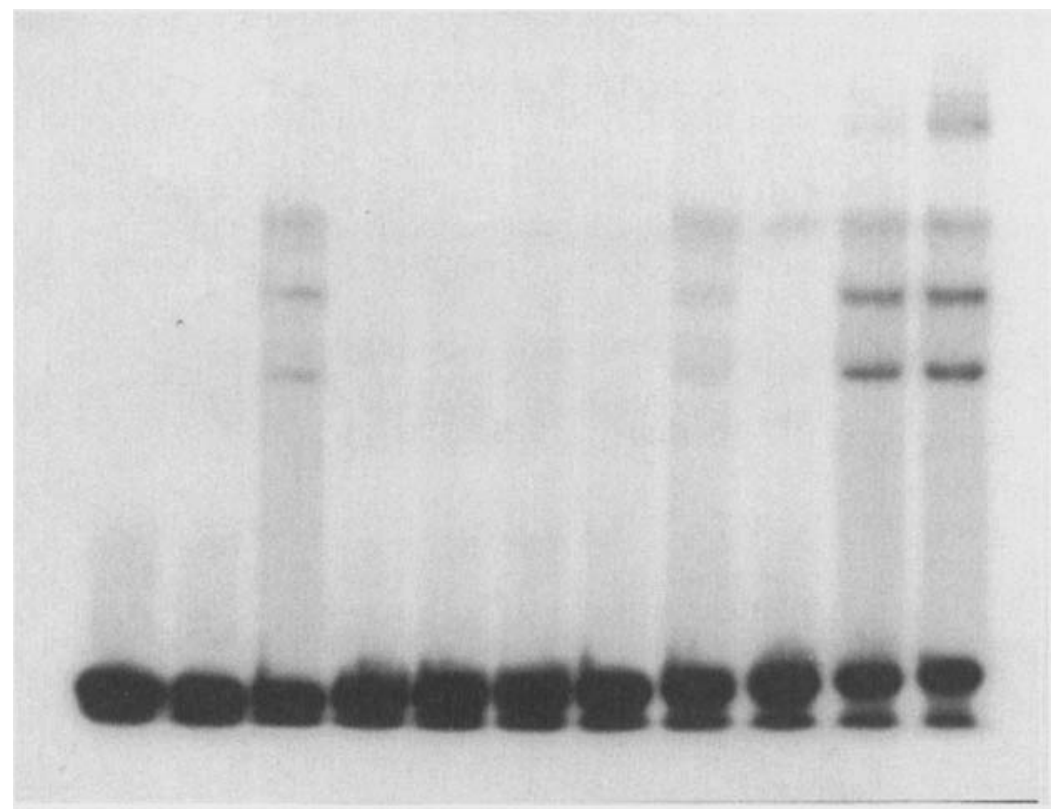

B.S.- CEA BllloligoEx1

FIGURE 2. Gel mobility shift assays were performed using c-erbA nuclear extracts (CEA). Gel retardation of an oligonucleotide corresponding to the putative TRE/RARE sequences (BIlloligoEx1), AGGAGCGCCCTGCACTGTGAGGTTCAAAC, was used as a labeled DNA fragment, in the presence or absence of positive oligonucleotide controls of previously characterized TREs (DIR2 and DIR4) and unspecific 38mer. P: labeled oligonucleotides without nuclear extract. WT: nuclear extract from wild type vaccinia infected HeLa cells. Numbers above the lanes indicate ng of cold oligonucleotides or $\mu$ of specific erbA antibodies (Ab) added.

have specific TRE) were added as competitors, the triplet was completely eliminated but not with unspecific oligonucleotide, $38 \mathrm{mer}$ ( $\mathrm{FIG}_{\mathrm{IG}}$ 2). The binding is specific, since the retardation complex was eliminated by competition by specific TRE but not by a 50-fold competitor with an unrelated sequence. These crosscompetition experiments confirm that the band 3 oligonucleotide contains a specific TRE sequence. Specific TR antibodies were used to supershift the retarded bands to confirm that the erbA receptors themselves are present in these DNAprotein complexes. The experiments with a mixture of TR and retinoic acid receptor suggest that the receptors bind to the band 3 gene as heterodimers (data not shown). 
These results strongly suggest that the v-erbA can arrest expression of the band 3 gene by direct binding to the regulatory region. Our finding of TRE/RARE within the band 3 gene is in line with the finding of the ability of v-erbA to repress retinoic acid receptor action and is closely correlated with its function in the establishment of the neoplastic phenotype. ${ }^{6}$ These experiments also raise the possibility that thyroid hormones and retinoids play an important role in regulation of band 3 gene expression.

\section{REFERENCES}

1. Zenke, M., A. Munoz, J. Sap, B. Vennström \& H. Beug. 1990. Cell 61: 1035-1049.

2. MCKAY, R. D. G. 1981. J. Mol. Biol. 145: 47I-488.

3. Stunnengerg, H., H. Lange, L. Phillipson, R. T. van Miltengurg \& P. C. van der VLIET. 1988. Nucleic Acids Res. 16: 2431-2444.

4. Kim, H. R. C., B. S. Kennedy \& J. D. EnGel. 1989. Molec. Cell Biol. 9: 5198-5206.

5. DeThe, H., M. D. M. Vivanco-Ruiz, P. Tiollais, H. Stunnenberg \& A. Dejean. 1990. Nature 343: 177-180.

6. Sande, S., M. Sharif, H. Chen \& M. Privalsky. 1993. J. Virol. 67: 1067-1074. 